\title{
UV-IRRADIATED HYDROGENATED AMORPHOUS CARBONS (HACS) AS CARRIERS OF THE INTERSTELLAR UV BUMP
}

\author{
K.A.K. Gadallah ${ }^{1,2}$, H. Mutschke ${ }^{2}$ and C. Jäger ${ }^{3}$
}

\begin{abstract}
Hydrogenated amorphous carbons (HACs) are considered as laboratory analogues to cosmic carbonaceous nanoparticles in the interstellar medium (ISM). The optical properties of nano-sized HACs may be influenced by the UV processing. The variation of the internal structure leads to dramatic changes in the spectral properties in the FUV-VIS range. This scenario can explain some astronomical features such as the interstellar UV bump at $4.6 \mu \mathrm{m}^{-1}$. The spectrum of HACs, irradiated by a dose of UV irradiation that corresponds to $21-33 \%$ of the average dose of the UV radiation in diffuse ISM, exhibits a new band centered at $4.6 \mu \mathrm{m}^{-1}$. This result confirms, for the first time, the suggestion by Mennella et al. (1996) that irradiated HACs might be considered as the carriers of the interstellar UV bump at $4.6 \mu \mathrm{m}^{-1}$. However, the amount of carbon needed to reproduce this band is higher than that available for interstellar carbon dust grains. So the ideal structure of irradiated HACs that would produce a band of sufficient strength has still to be searched for.
\end{abstract}

\section{Introduction}

In the diffuse interstellar medium (ISM), hydrogenated amorphous carbons (HACs) are bathed in a harsh UV radiation field (e.g., Ogmen \& Duley 1988). The radiation flux (F) is about $3 \times 10^{8} \mathrm{eV} \mathrm{cm}^{-2} \mathrm{~s}^{-1}$ hence the total dose (D) of UV photons deposited on HACs is $3 \times 10^{23} \mathrm{eV} \mathrm{cm}^{-2}$ during the lifetime $\left(3 \times 10^{7} \mathrm{yr}\right)$ of the diffuse ISM (Jenniskens 1993). The energy of UV photons is high enough to break $\mathrm{C}-\mathrm{H}$ bonds transforming HACs froming much more graphitic $\left(\mathrm{sp}^{2}\right)$ carbons.

1 Astronomy Dpt., Faculty of Science, Al-Azhar University, Nasr City, PO Box, 11884 Cairo, Egypt; e-mail: kamel.gadallah71@gmail.com

2 Astrophysical Institute and University Observatory, FSU, Schillergässchen 2/3, 07743 Jena, Germany

3 Institute of Solid State Physics, Friedrich Schiller University, Helmholtzweg 3, 07743 Jena, Germany 
The liberated energy could heat up the materials enough to remove $\mathrm{H}$ atoms before the recombination of some $\mathrm{H}$ atoms (Mennella et al. 2002) proceeds again. A remarkable feature of the interstellar extinction curve is the broad UV bump centered at $4.6 \mu \mathrm{m}^{-1}$ (Fitzpatrick 1999; Fitzpatrick \& Massa 1990). Its origin is still controversial since its discovery by Stecher (1965). Its peak has been found to be very stable at $4.6 \mu \mathrm{m}^{-1}$. Its carrier was thought to be small crystalline graphite particles (e.g., Draine \& Lee 1984). Laboratory (e.g., Jäger et al. 2008; Mennella et al. 1999) and theoretical studies (e.g., Duley \& Seahra 1998) suggested that the carriers of this band are hydrogenated carbonaceous solids, with submicron-sizes, including $\mathrm{CH}, \mathrm{CH}_{2}, \mathrm{CH}_{3}$ groups in connection with a graphitic backbone. Studies by Chhowalla et al. (2003); Mennella et al. (1998, 1996); Schnaiter et al. (1998); Wada et al. (1999) showed the importance of the electronic structure of HACs as a carrier of the UV bump. In addition to the polycyclic aromatic hydrocarbons (PAHs), which also contribute as carriers of this band (Allamandola et al. 1989), Mennella et al. (1996) attributed this band to the $\pi-\pi^{*}$ electronic transitions of the delocalized electrons in $\mathrm{sp}^{2}$ hybridized carbon bonds in carbon clusters. They found a band at $215 \mathrm{~nm}\left(4.65 \mu \mathrm{m}^{-1}\right)$ for UV-processed HACs with a dose of (1-4) $\times 10^{22} \mathrm{eV} \mathrm{cm} \mathrm{cm}^{2}$ corresponding to (3-13)\% of the average interstellar dose. To simulate closely the UV processing of HACs in the diffuse ISM, it is important to investigate spectroscopically the effect of higher values of UV doses. The production and the UV processing of the samples are shown in Section 2 while the results and discussion are given in Sections 3 and 4, respectively.

\section{Experiments}

The Nd:YAG laser ablation method (see Jäger et al. 2008, for more details) has been used to produce HAC samples from ablating a rotating graphite target in a quenching gas atomsphere $\left(\mathrm{He}: \mathrm{H}_{2}\right)$ at a pressure of 4.5 mbar. Under the physical conditions listed in Table 1 , the vibrational temperature of the plasma in the

Table 1. Physical conditions and analytical parameters of HAC samples produced in a gas pressure of 4.5 Torr.

\begin{tabular}{lcccccc}
\hline \hline S\# ${ }^{1}$ & $\begin{array}{c}\text { Atmosphere } \\
\left(\mathrm{He}: \mathrm{H}_{2}\right)\end{array}$ & $\begin{array}{c}F_{l} \\
\left(\mathrm{Wcm}^{-2}\right)\end{array}$ & $\begin{array}{c}\text { UV dose (Exp.) } \\
\left(\mathrm{eVcm}^{-2}\right)\end{array}$ & $\begin{array}{c}\text { \%D (ISM })^{(2)} \\
\left(\mathrm{eVcm}^{-2}\right)\end{array}$ & $\begin{array}{c}\mathrm{UV}^{-b u m p}{ }^{(3)} \\
\mu \mathrm{m}^{-1}\end{array}$ & $\begin{array}{c}N_{\mathrm{C}} / N_{\mathrm{H}}^{(4)} \\
\mathrm{ppm}\end{array}$ \\
\hline \hline $\mathrm{S} 4$ & $50: 30$ & $2 \times 10^{8}$ & $1.0 \times 10^{23}$ & 33 & 4.595 & 164 \\
$\mathrm{~S} 7$ & $50: 30$ & $2 \times 10^{8}$ & $1.0 \times 10^{23}$ & 33 & 4.607 & 269 \\
$\mathrm{~S} 8$ & $50: 30$ & $2 \times 10^{8}$ & $6.3 \times 10^{22}$ & 21 & 4.69 & 236 \\
$\mathrm{~S} 21$ & $90: 10$ & $2.0 \times 10^{10}$ & $6.3 \times 10^{22}$ & 21 & 4.639 & 108 \\
$\mathrm{~S} 23$ & $50: 20$ & $3.0 \times 10^{10}$ & $1.0 \times 10^{23}$ & 33 & 4.608 & 115 \\
\hline
\end{tabular}

${ }^{(1)} \mathrm{S} 8$ and $\mathrm{S} 23$ are deposited on a $\mathrm{CaF}_{2}$ substrate while the other samples are deposited on a $\mathrm{SiO}_{2}$ substrate. ${ }^{(2)}$ The corresponding percentage of the average interstellar dose. (3) The extracted UV bump position. ${ }^{(4)}$ The last column gives the required carbon abundance to match the band strength in the diffuse ISM. 


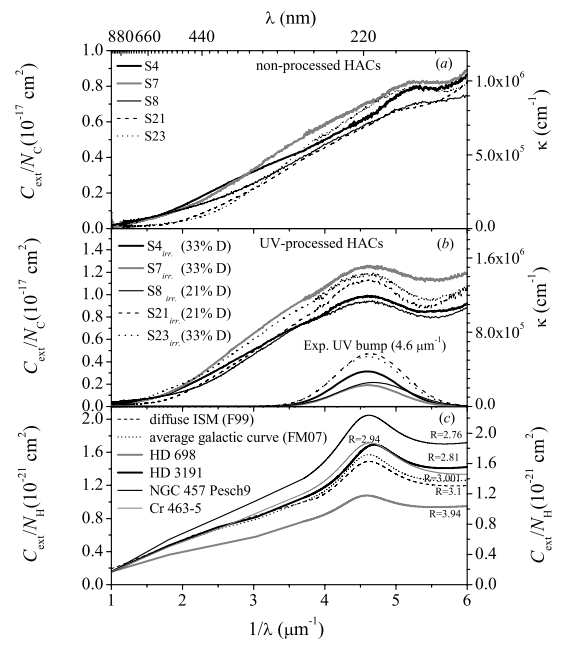

Figure 1. Panel (a) shows the spectra of non-processed samples while panel (b) shows the spectra of UV-processed samples in addition to the extracted UV bump around $4.6 \mu \mathrm{m}^{-1}$ by using Gaussian fitting. In panel $(c)$, the interstellar extinction curve of the diffuse ISM (Fitzpatrick 1999), the average galactic extinction curve, as well as extinction curves toward the stars HD 698, HD 3191, NGC 457 Pesch9 and Cr 463-5 (Fitzpatrick \& Massa 2007) are shown.

condensation zone is approximately $4000 \mathrm{~K}$ for samples (S4, S7, and S8) produced at using low laser power, $F_{l},\left(2 \times 10^{8} \mathrm{Wcm}^{-2}\right)$. While for samples (S21 and S23) produced at higher values of $F_{l}\left(2.0 \times 10^{10} \mathrm{Wcm}^{-2}\right.$ and $3.0 \times 10^{10} \mathrm{Wcm}^{-2}$, respectively), it rises to higher than $4000 \mathrm{~K}$ (Iida \& Yeung 1994). Subsequently a higher helium content, for the last two samples, is needed to confine the plasma by collisions to allow for an efficient condensation of solid HAC particles. Samples are deposited in a separate chamber at a pressure of $(5 \pm 3) \times 10^{-6}$ Torr on substrates of $\mathrm{SiO}_{2}$ and $\mathrm{CaF}_{2}$. Column 3 in Table 1 lists the UV doses that were applied on the samples in high vacuum $\left(\approx 3 \times 10^{-6}\right.$ mbar $)$ by using a discharge light source (deuterium lamp, L2D2 lamp, type L7292) that utilizes stable arc discharge in deuterium gas $\left(\mathrm{D}_{2}\right)$.

\section{Results and discussions}

The spectra in the FUV-NIR range of non-processed HACs are shown in panel (a) of Figure 1. The small peak appearing in the range from 5.0 to $5.3 \mu \mathrm{m}^{-1}$ was previously attributed to an additional $n-\sigma^{*}$ transition feature resulting from the attached $\mathrm{O}$-containing molecular groups such as $\mathrm{OH}$ groups (Llamas-Jansa et al. 2007). These groups could be due to the air exposure of the samples. It seems that this peak is very small and broad in the spectra of samples S21 and S23 produced with high laser power density, which leads to the formation of more C-H bonds and less porosities in HACs and consequently reduces the $\mathrm{O}-\mathrm{H}$ grouping. Panel $(b)$ shows a dramatic change in the spectra of samples after UV-processing. A big bump appears close to the $4.6 \mu \mathrm{m}^{-1}$. Its position is given in Table 1 for each sample. It comes very close to $4.6 \mu \mathrm{m}^{-1}$ with the higher UV doses while it appears at a slightely longer wavenumber with the lower UV doses. This indicates that the distinguishable UV band at $4.6 \mu \mathrm{m}^{-1}$ can be produced by the UV irradiation of carbonaceous nanoparticles in interstellar space as proposed by 
Mennella et al. (1996). Probably it results from $\pi-\pi^{*}$ electronic transitions due to the existence of the aromatic structures induced by the UV radiation within the HACs. According to studies by Cardelli et al. (1989), Fitzpatrick (1999, hereafter F99), and Fitzpatrick \& Massa (2007, hereafter FM07), the interstellar extinction consists of three components; the linear background, the Lorentzian-like $4.6 \mu \mathrm{m}^{-1}$ (217.5 nm) bump and the FUV non-linear rise. Figure 1 shows the extinction cross section per number of carbon atoms $\left(C_{\text {ext }} / N_{\mathrm{C}}\right)$ of the laboratory spectra of S4, S7, S8, S21 and S23 before $(a)$ and after (b) UV irradiation. In panel $(c)$, comparable curves of the extinction cross section per hydrogen atom $\left(C_{\text {ext }} / N_{\mathrm{H}}\right)$ for the average diffuse ISM (F99) and average galactic extinction curves as well as for some galactic objects (FM07) are shown. They are given for different values of the parameter $\mathrm{R}$ which represents the total-to- selective extinction ratio they are calculated by using the parameters taken from FM07 and from F99. The position of the $4.6 \mu \mathrm{m}^{-1}$ bump for laboratory UV-irradiated samples is in good agreement with that of the extinction curve of the diffuse ISM. However, high carbon abundances are required to match the strength of the diffuse ISM band (see Table 1). Similar values were derived by Schnaiter et al. (1998). The lack of carbon abundances in our results may be attributed to the inhomogeneity of the deepest irradiated materials, which are not completely irradiated.

\section{Conclusions}

HACs produced by laser ablation were irradiated by UV photons to simulate the interaction of the cosmic carbonaceous nanoparticles with UV radiation in the diffuse ISM. UV-processing in high vacuum with $21-33 \%$ of the average UV radiation dose in diffuse ISM produces a new band at $4.6 \mu \mathrm{m}^{-1}$ making irradiated HACs candidates for the carrier of this band in the interstellar extinction. However in order to reproduce the UV bump at $4.6 \mu \mathrm{m}^{-1}$ toward the diffuse ISM with a similar strength, the required amount of carbon based on our irradiated samples is between 108 and 269 ppm relative to hydrogen, which is higher than the carbon abundance available for interstellar dust grains. To solve this discrepancy, whole irradiated materials are needed to reduce the lack of carbons.

\section{References}

Allamandola, L.J., Tielens, A.G.G.M., \& Barker, J.R., 1989, ApJS, 71, 733

Cardelli, J.A., Clayton, G.C., \& Mathis, J.S., 1989, ApJ, 345, 245

Chhowalla, M., Wang, H., Sano, N., Teo, K.B., Lee, S.B., \& Amaratunga, G.A., 2003, Phys. Rev. Lett., 90, 155504

Draine, B.T., \& Lee, H.M., 1984, ApJ, 285, 89

Duley, W.W., \& Seahra, S., 1998, ApJ, 507, 874

Fitzpatrick, E.L., 1999, PASP, 111, 63

Fitzpatrick, E.L., \& Massa, D., 1990, ApJS, 72, 163

Fitzpatrick, E.L., \& Massa, D., 2007, ApJ, 663, 320 
Iida, Y., \& Yeung, E.S., 1994, Appl. Spectrosc., 48, 945

Jäger, C., Mutschke, H., Henning, T., \& Huisken, F., 2008, ApJ, 689, 249

Jenniskens, P., 1993, A\&A, 274, 653

Llamas-Jansa, I., Jger, C., Mutschke, H., \& Henning, T., 2007, Carbon, 45, 1542

Mennella, V., Brucato, J.R., Colangeli, L., \& Palumbo, P., 1999, ApJ, 524, L71

Mennella, V., Brucato, J.R., Colangeli, L., \& Palumbo, P., 2002, ApJ, 569, 531

Mennella, V., Colangeli, L., Bussoletti, E., Palumbo, P., \& Rotundi, A., 1998, ApJ, 507, L177

Mennella, V., Colangeli, L., Palumbo, P., Rotundi, A., Schutte, W., \& Bussoletti, E., 1996, ApJ, 464, L191

Ogmen, M., \& Duley, W.W., 1988, ApJ, 334, L117

Schnaiter, M., Mutschke, H., Dorschner, J., Henning, T., \& Salama, F., 1998, ApJ, 498, 486

Stecher, T.P., 1965, ApJ, 142, 1683

Wada, S., Kaito, C., Kimura, S., \& Tokunaga, A.T., 1999, Adv. Space Res., 24, 523 